\title{
Use of Additive Manufacturing on Models for Sand Casting Process
}

\author{
C. Bermudo, S. Martín-Béjar, F.J. Trujillo, L. Sevilla \\ Department of Manufacturing Engineering, University of Malaga, C/Dr. Ortiz Ramos s/n, E-29071 Malaga, Spain \\ * Corresponding author: Tel.: +34-951-952-245; E-mail address: bgamboa@uma.es
}

Topic classification: Additive Manufacturing

Keywords: Additive Manufacturing; Sand Casting; Permanent Model; Expendable Mold

\begin{abstract}
Casting is a forming process based on material pouring in liquid state. Heat is applied to melt the material from its solid state and pour it in a mold previously constructed to obtain the desired shape after letting it cool. Molds can be permanent or expendable. In sand casting processes, the mold is manufactured from a model that usually is extracted before pouring the melted material (Figure 1). To obtain the part, the sand model needs to be destroyed. Notwithstanding, the sand can be reused several times for new molds [1].
\end{abstract}

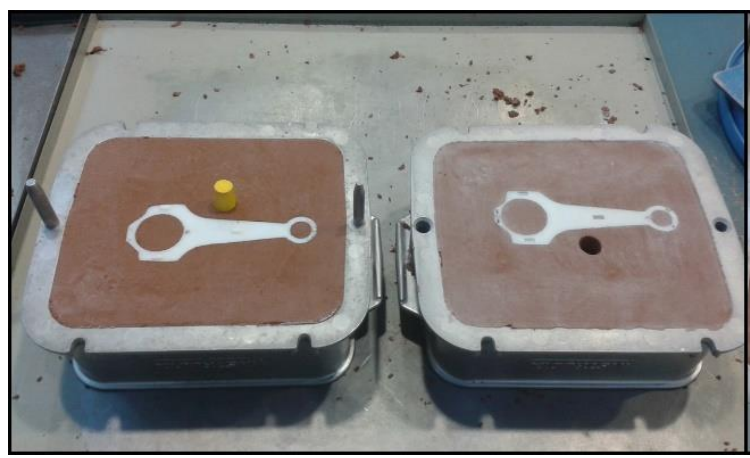

(a)

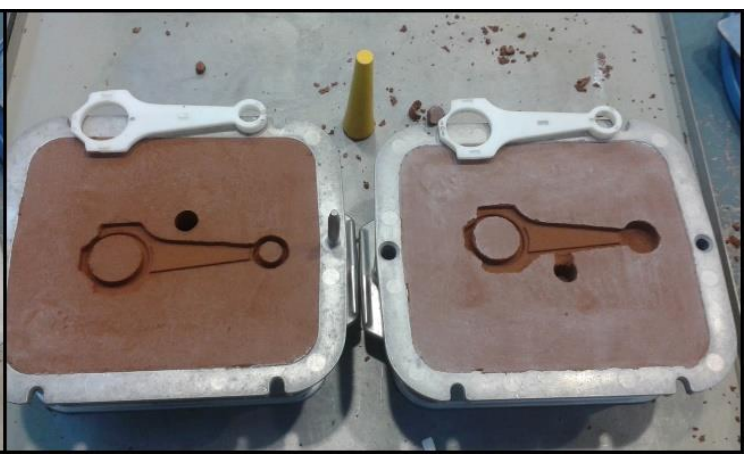

(b)

Figure 1: Sand casting process. Open mold with patterns and sprue inserted (a) and mold with pattern extracted ready to be closed $(b)$

Several elements are needed to obtain a part by a sand casting process: permanent patterns, flasks (cope and drag), gating system (pouring cup, sprue, risers and feeding channels) and cores (only if it is needed). The aim of this work is the manufacturing of some of these elements by additive manufacturing process. The equipment will be used for the practical activities (Figure 1) that currently take place on the subjects where this topic is taught, in the different Degrees in Industrial Engineering at the University of Malaga.

These elements were manufactured using an additive manufacturing technique, specifically a Fused Deposition Modeling (FDM) process. The main advantages of the additive process versus the conventional procedures (machining, polymer injection molding, etc.) are that allows complex shapes, the pattern can be reprinted as many times as desired and, in addition, it is an economical process [2]. Notwithstanding, there are negative points to take into account: low resolution (finishing operations are needed) and low strength, among others.

The parts obtained for this work are not intended for a direct industrial application but, with the appropriate scale and a higher 3D printer resolution, the methodology exposed can be implemented in an industrial environment.

First, a selection of the pattern shapes is made, taking into account the actual shapes and the future requirements. The designs are obtained using the software SolidWorks. This application helps the designer to allocate the necessary draft angle in the virtual part, in order to be able to remove the pattern and, later, the real part from the mold. If the angles are not well designed, the mold will suffer crumbling that may affect the final aspect of the part and, in worst cases, could make impossible the casting process. In order to build the optimal pattern shape, an approximation to the different analytical methods of calculation for the sand casting elements is made, Figure 1a [3,4]. So, the gating and feeding system are calculated to ensure the correct filling of the mold (Table 1). For this purpose, different simulations with SOLIDCast 8.0 are 
carried out (Figure 2a), being $D_{1}$ and $D_{2}$ the sprue upper and lower diameter, $D_{\text {sprue base }}$ the sprue base diameter, $h_{\text {sprue base }}$ the sprue base height and $e_{c}$ the pouring cup thickness.

Table 1: Sizing results of the cast gating system. Analytical method

\begin{tabular}{cccccc}
\cline { 2 - 6 } & $\begin{array}{c}\boldsymbol{D}_{\mathbf{1}} \\
(\mathbf{m m})\end{array}$ & $\boldsymbol{D}_{\mathbf{2}}(\mathbf{m m})$ & $\boldsymbol{D}_{\text {sprue base }}(\mathbf{m m})$ & $\boldsymbol{h}_{\text {sprue base }}(\mathbf{m m})$ & $\boldsymbol{e}_{\mathbf{c}}(\mathbf{m m})$ \\
\hline Connecting rod & 5.973 & 2.726 & 5.453 & 4.275 & 2.138 \\
Crankshaft & 5.516 & 2.518 & 5.035 & 3.948 & 1.974 \\
Piston & 5.384 & 2.458 & 4.915 & 3.854 & 1.927 \\
Anvil & 8.314 & 3.795 & 7.590 & 5.951 & 2.975 \\
Generic sprue & 25 & 14.05 & 12 & 15 & 8 \\
\hline
\end{tabular}

Then, the manufacturing process of the different selected patterns is carried out with an additive process. Due to the low resolution of the 3D printer available, the surface integrity of the patterns usually damages the sand mold when is extracted. Hence, to avoid this problem, a finishing operation is performed on the patterns surface (Figure 2b). In addition, different systems are designed and implemented to make the pattern extraction easier (Figure 2c).

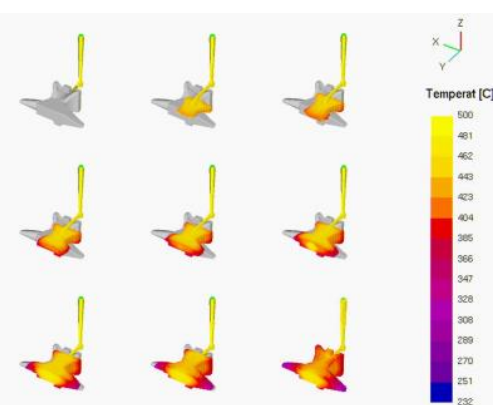

(a)

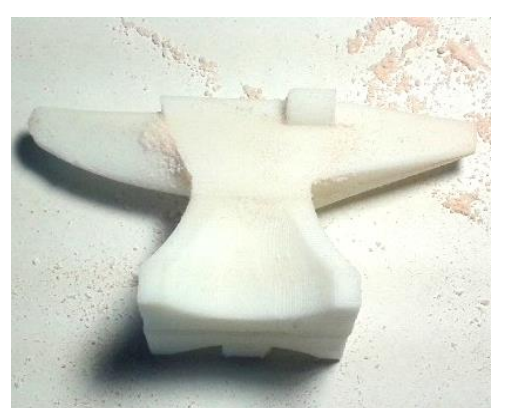

(b)

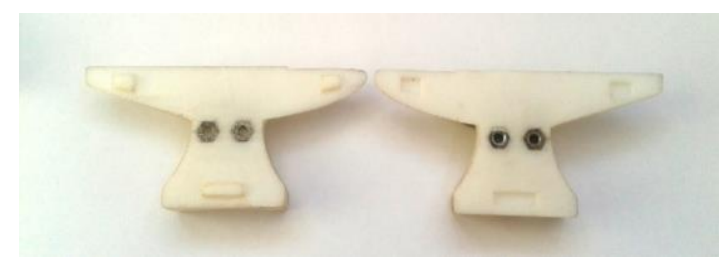

(c)

Figure 2: 3D printed anvil pattern after surface finishing process (a), pouring and filling mold simulation with an optimal sprue (b) and extraction system simulation (c)

In conclusion, the implementation of the additive process fulfills the aims of this work. Additionally, the total cost of the materials used for this study (printing only one model of each pattern) is of $46.00 €$. This makes the additive process a good alternative to current more expensive techniques (machining, polymer molding, etc.). Also, this budget makes feasible the implementation of the additive processes in order to obtain more patterns and any necessary equipment for the sand casting processes.

\section{Acknowledgements}

The authors thank University of Malaga - Andalucía Tech Campus of International Excellence for its contribution on this paper

\section{References}

1. Kalpakjian, S.; Schmid, S. R. Manufacturing Engineering and Technology.; 7th ed.; Pearson: New Jersey (USA), 2014; ISBN 978-0133128741.

2. Upadhyay, M.; Sivarupan, T.; El Mansori, M. 3D printing for rapid sand casting-A review. J. Manuf. Process. 2017, 29, 211-220, doi:10.1016/J.JMAPRO.2017.07.017.

3. Wang, W.; Stoll, H. W.; Conley, J. G. Rapid tooling guidelines for sand casting; Springer, 2010; ISBN 1441957316.

4. Shangguan, H.; Kang, J.; Deng, C.; Hu, Y.; Huang, T. 3D-printed shell-truss sand mold for aluminum castings. J. Mater. Process. Technol. 2017, 250, 247-253, doi:10.1016/J.JMATPROTEC.2017.05.010. 